\title{
Association of Serum Uric Acid, Urea Nitrogen, and Urine Specific Gravity Levels at 16-18 Weeks of Gestation with the Risk of Gestational Diabetes Mellitus
}

This article was published in the following Dove Press journal:

Diabetes, Metabolic Syndrome and Obesity: Targets and Therapy

\author{
Yan $\mathrm{Li}^{1}{ }^{1} *$ \\ Tingwei $\mathrm{Yu}^{2, *}$ \\ Zengyou Liu $^{2}$ \\ Hengying Chen ${ }^{3}$ \\ Yao Liu $^{4}$ \\ Yuanhuan $\mathrm{Wei}^{4}$ \\ Ruifang Sun ${ }^{4}$ \\ Hongmei Zhang ${ }^{2}$ \\ Wei Wang ${ }^{2}$ \\ Yihua $\mathrm{Lu}^{2}$ \\ Yingyu Zhou' \\ Guifang Deng ${ }^{4}$ \\ Zheqing Zhang
}

'Department of Nutrition and Food Hygiene, Guangdong Provincial Key Laboratory of Tropical Disease Research, School of Public Health, Southern Medical University, Guangzhou, People's Republic of China; ${ }^{2}$ Department of Obstetrics, Union Shenzhen Hospital of

Huazhong University of Science and Technology, Shenzhen, People's Republic of China; ${ }^{3}$ Injury Prevention Research Center, Shantou University Medical College, Shantou, People's Republic of China; ${ }^{4}$ Department of Clinical Nutrition, Union Shenzhen Hospital of Huazhong University of Science and Technology, Shenzhen, People's Republic of China

*These authors contributed equally to this work

Correspondence: Zheqing Zhang

Department of Nutrition and Food Hygiene,

Guangdong Provincial Key Laboratory of

Tropical Disease Research, School of Public

Health, Southern Medical University, No. 1023-

1063, Shatai South Road, Baiyun District,

Guangzhou, Guangdong 510515, People's

Republic of China

Email zzqaa501@smu.edu.cn

Guifang Deng

Department of Clinical Nutrition, Union

Shenzhen Hospital of Huazhong University of

Science and Technology, No. 89 Taoyuan Road,

Shenzhen, Guangdong 518052, People's

Republic of China

Email misyfly@I63.com
Objective: To evaluate the associations of serum uric acid (UA), urea nitrogen (UN), and urine specific gravity (USG) levels in the first trimester of pregnancy with the risk of gestational diabetes mellitus (GDM).

Patients and Methods: A retrospective cohort study was conducted in 1,769 pregnant women aged $31.55 \pm 3.91$ years. UA, UN, and USG levels were measured during the 1618 th week of gestation. GDM was diagnosed by an oral $75 \mathrm{~g}$ glucose tolerance test during the 24-28th week of gestation.

Results: A multivariate adjusted logistic regression analysis showed that UA levels in the highest quartile increased the risk of GDM by 55.7\% (odds ratio [OR]: $1.557,95 \%$ confidence interval $[\mathrm{CI}]: 1.055-2.298 ; p=0.026)$ compared to those in the lowest quartile. USG levels in the second, third, and fourth quartiles increased the risk of GDM by $67.6 \%$ (95\% CI: $1.090-2.421), 112.4 \%$ (95\% CI: $1.446-3.119)$, and 94.5\% (95\% CI: $1.314-2.880)$, respectively, compared to those in the first quartile $(p$ trend $=0.001)$. No significant association between UN levels and the GDM risk was observed. When the extreme composite biomarker score quartiles were compared, the adjusted OR (95\% CI) for GDM was 1.909 (95\% CI: 1.332-2.736). Age-stratified analyses revealed similar results in women aged $\leq 35$ years only, but not in those aged $>35$ years.

Conclusion: Higher levels of UA and USG and a higher composite kidney function biomarker score during the 16-18th week of gestation were positively and independently associated with an increased risk of GDM.

Keywords: uric acid, urea nitrogen, urine specific gravity, gestational diabetes mellitus, retrospective cohort study

\section{Introduction}

Gestational diabetes mellitus (GDM) is defined as any degree of glucose intolerance with an onset during pregnancy. ${ }^{1}$ The World Health Organization (WHO) reported that the global prevalence of GDM was between 5\% and 13\% from 2005 to 2015 . $^{2}$ A recent meta-analysis including 79,064 pregnant women from the general population revealed that the prevalence of GDM in mainland China was $14.8 \%{ }^{3}$ An elevated blood glucose level during pregnancy is associated with adverse short- and long-term outcomes both for the mothers and their offspring. ${ }^{4-11}$ Thus, identification of the associated risk factors is urgently required to prevent GDM. 
Uric acid (UA), urea nitrogen (UN), and urine specific gravity (USG) are important indicators of kidney function. ${ }^{12-14}$ Previous studies have reported that the serum UA level is associated with insulin resistance in nonpregnant women ${ }^{15}$ and is a strong independent risk factor for type 2 diabetes mellitus (T2DM). ${ }^{16}$ Although studies have explored the relationship between the blood UA level and GDM, the findings have been inconsistent. An elevated serum UA level has been previously reported to be a risk factor for GDM by some studies ${ }^{17,18}$ but not by others. ${ }^{19} \mathrm{UN}$ is another indicator of renal function and has been demonstrated to promote reactive oxygen species (ROS) generation and subsequent insulin resistance in mouse models. ${ }^{20} \mathrm{~A}$ recent large prospective cohort study of 1,337,452 United States Veterans suggested that every $10 \mathrm{mg} / \mathrm{dL}$ increase in the blood UN (BUN) concentration resulted in a $15 \%$ higher risk of developing diabetes. ${ }^{21}$ To date, only one study has explored whether UN levels in the first trimester are related to the risk of developing GDM, and identified $\mathrm{UN}$ as a potential predictor of GDM. ${ }^{22}$ However, whether UN is associated with GDM remains to be fully determined.

USG is defined as the ratio of the weight of the urine to that of an equal volume of water. ${ }^{23}$ In animal models, higher urea levels led to increased islet protein O-GlcNAcylation and impaired glycolysis, and ultimately led to insulin secretion defects during chronic kidney disease. ${ }^{14}$ However, no previous human studies have determined the correlation between USG and GDM. Because the nature of the associations of UA, UN, and USG with GDM is unclear, further studies are warranted. Therefore, we sought to explore whether UA, UN, USG, and the combination of these biomarkers (ie, a composite biomarker score) are linked to GDM in a retrospective cohort study.

\section{Patients and Methods}

\section{Subject}

A total of 1,836 pregnant women who registered at the Union Shenzhen Hospital of Huazhong University of Science and Technology (Shenzhen, Guangdong) and planned to deliver their child at this hospital were recruited from January 2015 to December 2018. Of these, 67 mothers were excluded for the following reasons: history of diabetes $(n=9)$, liver or kidney disease $(n=44)$, heart disease $(\mathrm{n}=5)$, hypertension $(\mathrm{n}=1)$, and twin pregnancy $(n=9)$. Elevated levels of UA and a higher prevalence of
GDM in twin pregnancies, compared with those in their singleton counterparts, have been reported previously. ${ }^{24,25}$ Thus, the inclusion of twin pregnancies may overestimate the relationship between GDM and the chosen markers of kidney function. Therefore, twin pregnancies were excluded from the study $(\mathrm{n}=9)$. In total, 1,769 women aged 20-45 years with singleton pregnancies were included in this study. All women were screened for GDM based on the 2010 diagnostic criteria of the International Association of Diabetes and Pregnancy Study Group (IADPSG) ${ }^{26}$ In brief, GDM was diagnosed if the fasting blood glucose levels reached $5.1 \mathrm{mmol} / \mathrm{L}$ or if glucose levels reached $10 \mathrm{mmol} / \mathrm{L}$ within 1 hour or 8.5 $\mathrm{mmol} / \mathrm{L}$ within 2 hours of an oral glucose tolerance test (OGTT). The OGTT was performed using a one-step method during the 24-28th week of pregnancy. All of the participants provided informed consent to take part in this study. This study was approved by the Ethics Committee of the Union Shenzhen Hospital of Huazhong University of Science and Technology and complied with the ethical guidelines set forth by the World Medical Association Declaration of Helsinki (No. 2019072644).

\section{Basic Information Collection}

A novel questionnaire was used to obtain information about each subject. Age, education, smoking status, alcohol status, conception method, parity, embryo number, and history of disease (eg, diabetes, liver or kidney disease, heart disease, and hypertension) were collected through face-to-face interviews. The heights and weights of the participants were measured every $\sim 6$ weeks during the pregnancy using a height and weight scale accurate to $0.1 \mathrm{~cm}$ and $0.1 \mathrm{~kg}$, respectively. Pre-pregnancy body mass index (BMI) was calculated as the weight $(\mathrm{kg})$ divided by the height squared $\left(\mathrm{m}^{2}\right)$.

\section{Measurement of Uric Acid, Urea Nitrogen, and Urine Specific Gravity}

During the 16-18th week of gestation, fasting venous blood and urine samples were collected for further analyses. The samples were centrifuged at $3,500 \mathrm{rpm}$ at $4^{\circ} \mathrm{C}$ for 5 minutes within 2 hours of collection. Serum concentrations of UA and UN were determined using a colorimetric assay with an ACCELERATOR a3600 automatic analyzer (Abbott, Chicago, USA), while the USG was determined enzymatically using a Hitachi 7600 automatic analyzer (Hitachi, Tokyo, Japan). A composite 
biomarker score was generated by calculating the standardized values of biomarkers which exhibited significant association with the prevalence of GDM. The coefficients of variation for the mixed samples were $1.29 \%$ for UA, $2.34 \%$ for UN, and $1.05 \%$ for USG.

\section{Statistical Analysis}

Values are presented as means \pm standard deviations for continuous variables and as frequencies (\%) for categorical variables. Differences between the GDM and normal groups were tested using Student's $t$-test for continuous variables, while the chi-square test was used for categorical variables. The UA, UN, and USG values and the composite biomarker scores were divided into quartiles. Logistic regression analyses were performed to calculate the odds ratios (ORs) and 95\% confidence intervals (95\% CIs) for the risk of GDM across each of the quartiles. Model 1 was a univariate analysis, while model 2 was adjusted for age, pre-pregnancy BMI, education, smoking status, alcohol status, parity, and conception method. Advanced maternal age is a known risk factor for GDM. $^{27}$ A meta-analysis demonstrated that the magnitude of the correlation of UA with T2DM varied with age. ${ }^{16}$ Hence, we performed a subgroup analysis by age $(\leq 35$ and $>35$ years). Statistical analyses were conducted using IBM SPSS, version 22.0. A two-sided $p$-value $<0.05$ was considered statistically significant.

\section{Results}

A total of 1,769 women aged $31.55 \pm 3.91$ years were included in this study (Table 1). Mothers diagnosed with GDM were older $(32.37 \pm 3.95$ vs $31.31 \pm 3.86, p<0.001)$ and had a higher pre-pregnancy BMI $(21.80 \pm 2.71$ vs $20.61 \pm 3.39, p<0.001)$, UA $(216.45 \pm 46.07 \mu \mathrm{mol} / \mathrm{L}$ vs $209.36 \pm 42.87 \mu \mathrm{mol} / \mathrm{L}, p=0.007)$, and USG (1.017 \pm 0.005 vs $1.016 \pm 0.005, p<0.001)$ than those with normal glucose levels. No significant group differences were detected in terms of education, smoking status, alcohol status, conception method, parity, or UN level $(p>0.05)$.

Table 2 shows the ORs (95\% CIs) for GDM according to the UA levels. After adjustment for age, pre-pregnancy BMI, education, smoking status, alcohol status, parity, and conception method, a dose-response relationship between the UA levels and the risk of GDM was observed. Women with UA levels in the fourth quartile had a $46.3 \%(\mathrm{OR}=$ 1.463, 95\% CI: 1.034-2.070) higher risk of GDM than those in the first quartile. After stratification by age, a multivariate analysis revealed that among women aged $\leq 35$ years, those with UA levels in the highest quartile had a 55.7\% $(\mathrm{OR}=1.557,95 \% \quad \mathrm{CI}: 1.055-2.298)$ increased risk of GDM relative to those in the lowest quartile. No significant group differences across quartiles were observed among women $>35$ years old.

The relationship between UN concentration and GDM incidence is shown in Table 3. After adjusting for confounding factors, no significant relationship was observed between UN levels and the GDM risk at 16-18 weeks of gestation. Similar results were observed in the agestratified analyses.

As seen in Table 4, a dose-response relationship was observed between the USG at 16-18 weeks of gestation and the risk of GDM. The multivariable-adjusted ORs (95\% CIs) across the quartiles of USG were 1 (reference), 1.741 (1.233-2.458; second quartile), 1.863 (1.319-2.633; third quartile), and 1.703 (1.203-2.411; fourth quartile) ( $p$ trend $=0.001$ ). When stratified by age, women aged $\leq$ 35 years with USG in the fourth quartile had a two-fold higher risk of developing GDM (OR: 1.945, 95\% CI: 1.314-2.880) compared to those in the first quartile.

Table 5 shows the ORs (95\% CIs) for GDM according to the quartiles of the composite biomarker score calculated by the standardized values of UA and USG. The multivariable-adjusted ORs (95\% CIs) across the quartiles of the composite biomarker score were 1 (reference), 1.342 (0.926-1.945; second quartile), 1.681 (1.171-2.413; third quartile), and 1.909 (1.332-2.736; fourth quartile; $p$ trend $=0.003)$. Stratified analyses indicated that the positive association between composite biomarker scores and GDM remained significant only in subjects aged $\leq 35$ years, but not in those aged $>35$ years.

\section{Discussion}

In this retrospective cohort study investigating the risk factors for GDM, we observed that pregnant women with increased levels of UA and USG during the 16-18th week of gestation exhibited a higher risk of developing GDM. No significant relationship between UN levels and the GDM risk was observed.

In vitro studies have suggested that elevated UA levels might induce ROS production, which leads to insulin resistance and decreased glucose uptake. ${ }^{20,28}$ Additionally, uric acid-mediated endothelial cell dysfunction reduces nitric oxide (NO) production. ${ }^{29}$ Roy et al demonstrated that insulin-regulated glucose uptake by muscle cells and adipocytes depends on NO. ${ }^{30}$ A metaanalysis involving 32,016 participants provided strong 
Table I Characteristics of the Participants in This Study $(\mathrm{N}=1769)$

\begin{tabular}{|c|c|c|c|}
\hline Characteristics & $\begin{array}{l}\text { Normal } \\
(n=1373)\end{array}$ & $\begin{array}{l}\text { GDM } \\
(n=396)\end{array}$ & $P$ \\
\hline Age (years) & $31.31 \pm 3.86$ & $32.37 \pm 3.95$ & $<0.001$ \\
\hline Age categories n (\%) & & & \\
\hline$\leq 35$ & II 57 (84.27) & $311(78.54)$ & 0.007 \\
\hline$>35$ & $216(15.73)$ & $85(21.46)$ & \\
\hline $\begin{array}{l}\text { Pre-pregnancy BMI } \\
\left(\mathrm{kg} / \mathrm{m}^{2}\right)\end{array}$ & $20.61 \pm 3.39$ & $21.80 \pm 2.71$ & $<0.001$ \\
\hline \multicolumn{4}{|l|}{ OGTT (mmol/L) } \\
\hline FPG & $4.52 \pm 0.26$ & $4.90 \pm 0.45$ & $<0.001$ \\
\hline I hour & $7.48 \pm 1.32$ & $9.80 \pm 1.61$ & $<0.001$ \\
\hline 2 hour & $6.54 \pm 0.97$ & $8.57 \pm 1.47$ & $<0.001$ \\
\hline \multicolumn{4}{|l|}{ Education n (\%) } \\
\hline Primary & $45(3.28)$ & $14(3.54)$ & \\
\hline Secondary & $202(\mid 4.7 I)$ & 55 (13.89) & 0.897 \\
\hline College or above & $1126(82.01)$ & $327(82.57)$ & \\
\hline \multicolumn{4}{|l|}{ Smoking status n (\%) } \\
\hline Yes & $2(0.07)$ & 0 & \\
\hline No & I 325 (96.58) & 391 (97.47) & 0.614 \\
\hline NA & $46(3.35)$ & $10(2.53)$ & \\
\hline \multicolumn{4}{|l|}{ Alcohol status n (\%) } \\
\hline Yes & $2(0.15)$ & $\mathrm{I}(0.25)$ & \\
\hline No & $1330(96.50)$ & 385 (97.22) & 0.643 \\
\hline NA & $46(3.35)$ & $10(2.53)$ & \\
\hline \multicolumn{4}{|c|}{ Conception method n (\%) } \\
\hline Natural & 1345 (97.96) & $385(97.22)$ & \\
\hline Artificial & $14(1.02)$ & $8(2.02)$ & 0.258 \\
\hline NA & $14(1.02)$ & $3(0.76)$ & \\
\hline \multicolumn{4}{|l|}{ Parity n (\%) } \\
\hline Primiparity & 615 (44.79) & I8I (45.7I) & 0.747 \\
\hline Multiparity & $758(55.2 I)$ & $215(54.29)$ & \\
\hline Uric Acid (umol/L) & $209.36 \pm 42.87$ & $216.45 \pm 46.07$ & 0.007 \\
\hline $\begin{array}{l}\text { Urea nitrogen (mmol/ } \\
\text { L) }\end{array}$ & $2.623 \pm 0.628$ & $2.627 \pm 0.579$ & 0.907 \\
\hline Urine specific gravity & $1.016 \pm 0.005$ & $1.017 \pm 0.005$ & $<0.001$ \\
\hline
\end{tabular}

Note: Values are Mean \pm SD for continuous variables and frequencies (percentages) for categorical variables.

Abbreviations: GDM, gestational diabetes mellitus; BMI, body mass index; OGTT, oral glucose tolerance test; FPG, fasting plasma glucose; NA, not available.

evidence that high serum UA levels were positively associated with the development of T2DM. ${ }^{16}$ Only a few studies have evaluated the association of UA levels with GDM. During a normal pregnancy, UA concentrations were found to decrease significantly by the 8 th week of gestation, compared to pre-pregnancy levels, and these reduced levels were maintained until approximately 24 weeks of gestation. ${ }^{31}$ In a prospective study including 1,570 subjects, Laughon et al found that the age- and BMIadjusted risk of GDM increased 3.25-fold (95\% CI, 1.35-7.83) in women with UA levels in the highest quartile during the first trimester relative to that in women with UA levels in the lowest quartile. ${ }^{32}$ In a retrospective analysis of 626 subjects in Turkey, Şahin et al observed that an elevated serum level of UA in early pregnancy was positively associated with the risk of GDM in the second trimester. ${ }^{17}$ Similarly, Wolak et al reported that UA levels in the fourth quartile detected during the 20th week of pregnancy were associated with a higher incidence of GDM among Egyptian pregnant women. ${ }^{18}$ In contrast, Güngör et $\mathrm{al}^{19}$ and Maged et $\mathrm{al}^{33}$ reported that serum UA concentrations play no role in GDM development. These findings from previous studies are controversial, and no studies, until now, have been conducted in China. Previous studies have demonstrated that ethnicity potentially modifies the relationship between UA and metabolic syndromes. ${ }^{34}$ We extended these findings in a relatively large cohort of pregnant Chinese women and observed that women with UA levels in the fourth quartile during the 16-18th week of pregnancy exhibited a $46.3 \%$ higher risk of GDM at 24-28 weeks. The heterogeneous nature of the results reported in previous studies may be due to variation in study design, sample size, biomarker evaluation time points, diagnostic criteria, or other confounding factors.

A meta-analysis involving 32,016 participants reported that the association between UA and T2DM was stronger in the younger subgroup $(<50$ years $)$ than in the older subgroup ( $\geq 50$ years). ${ }^{16}$ Pathophysiological changes associated with aging may provide a potential explanation for these age-related differences, as aging is an inevitable risk factor for developing insulin resistance. ${ }^{35}$ If elevated UA levels can lead to insulin resistance, changes in UA levels may not affect older pregnant women who have already developed insulin resistance due to natural aging. We also performed interaction and age-stratified analyses to evaluate whether age modifies the relationship between these kidney function markers and GDM. We found a significant correlation between UA levels and GDM development in women aged $\leq 35$ years, but not in women aged $>35$ years. However, there is no evidence of a statistically significant interaction between age and kidney function biomarkers ( $p$ : 0.231-0.680), suggesting that this correlation is not due to a causal relationship. Further studies with 
Table 2 ORs $(95 \% \mathrm{Cl})$ for the Occurrence of GDM According to the Quartiles of Uric Acid ( $N=1579)$

\begin{tabular}{|c|c|c|c|c|c|}
\hline & \multicolumn{4}{|c|}{ Quartiles of Uric Acid } & \multirow[t]{2}{*}{$P$ Trend } \\
\hline & QI & Q2 & Q3 & Q4 & \\
\hline \multicolumn{6}{|l|}{ Total } \\
\hline N (case/control) & $74 / 320$ & $91 / 305$ & $84 / 307$ & $108 / 290$ & \\
\hline Model I & I & $1.290(0.914,1.985)$ & I.183 (0.834, I.678) & $1.610(1.151,2.253)$ & 0.042 \\
\hline Model 2 & I & I.195 (0.840, I.699) & I.III (0.777, I.588) & $1.463(1.034,2.070)$ & 0.051 \\
\hline \multicolumn{6}{|c|}{ Stratified analysis by age } \\
\hline \multicolumn{6}{|l|}{$\leq 35$ years } \\
\hline $\mathrm{N}$ (case/control) & $57 / 268$ & $72 / 255$ & $63 / 261$ & $86 / 241$ & \\
\hline Model I & I & $1.328(0.90 \mathrm{I}, \mathrm{I} .956)$ & I.I35 (0.763, I.688) & I.678 (I.15I, 2.447) & 0.040 \\
\hline Model 2 & I & $1.210(0.814,1.798)$ & $1.042(0.694,1.564)$ & $1.557(1.055,2.298)$ & 0.054 \\
\hline \multicolumn{6}{|l|}{$>35$ years } \\
\hline $\mathrm{N}$ (case/control) & $17 / 52$ & $19 / 50$ & $21 / 46$ & $22 / 49$ & \\
\hline Model I & I & I. $162(0.543,2.487)$ & $1.396(0.658,2.964)$ & $1.373(0.653,2.889)$ & 0.799 \\
\hline Model 2 & I & I. $147(0.529,2.483)$ & $\mathrm{I} .405(0.65 \mathrm{I}, 3.034)$ & $\mathrm{I} .697(0.538,2.529)$ & 0.857 \\
\hline
\end{tabular}

Notes: Model I, without adjustment; Model 2, adjustment for age, pre-pregnancy BMI, education, smoking status, alcohol status, parity, conception method. Abbreviation: GDM, gestational diabetes mellitus.

larger sample sizes are required to clarify the relationship between UA levels and the GDM risk and how this is influenced by age.

$\mathrm{UN}$, which is generally recognized to be a biomarker of kidney function, has recently received attention because of its association with insulin resistance ${ }^{14}$ and diabetes. ${ }^{21}$ Previous experimental studies, both in vitro and in vivo, have demonstrated that elevated urea levels can promote ROS generation, induce low-grade inflammation, and thus elicit insulin resistance and inhibit insulin secretion. ${ }^{20,36}$ A prospective cohort study of United States Veterans provided evidence to support the hypothesis that higher UN levels are associated with an increased risk of developing diabetes mellitus $(\mathrm{DM})^{21}$ and an increased likelihood of

Table 3 ORs $(95 \% \mathrm{Cl})$ for the Occurrence of GDM According to the Quartiles of Urea Nitrogen $(\mathrm{N}=1576)$

\begin{tabular}{|c|c|c|c|c|c|}
\hline & \multicolumn{4}{|c|}{ Quartiles of Urea Nitrogen } & \multirow[t]{2}{*}{$P$ Trend } \\
\hline & QI & Q2 & Q3 & Q4 & \\
\hline \multicolumn{6}{|l|}{ Total } \\
\hline $\mathrm{N}$ (case/control) & $95 / 341$ & $96 / 262$ & $71 / 303$ & $93 / 315$ & \\
\hline Model I & I & $1.315(0.949,1.822)$ & $0.84 \mid(0.596, I .187)$ & $1.060(0.766,1.466)$ & 0.087 \\
\hline Model 2 & I & $1.334(0.956,1.863)$ & $0.904(0.637,1.284)$ & $1.149(0.823,1.604)$ & 0.941 \\
\hline \multicolumn{6}{|c|}{ Stratified analysis by age } \\
\hline \multicolumn{6}{|l|}{$\leq 35$ years } \\
\hline $\mathrm{N}$ (case/control) & $74 / 293$ & $72 / 210$ & $54 / 258$ & $76 / 264$ & \\
\hline Model I & I & $1.358(0.938,1.965)$ & $0.829(0.562,1.222)$ & I.I $40(0.795,1.635)$ & 0.092 \\
\hline Model 2 & I & $1.403(0.962,2.047)$ & $0.869(0.585,1.292)$ & $1.254(0.864,1.819)$ & 0.077 \\
\hline \multicolumn{6}{|l|}{$>35$ years } \\
\hline $\mathrm{N}$ (case/control) & $21 / 48$ & $24 / 52$ & $17 / 45$ & $|7 / 5|$ & \\
\hline Model I & I & $\mathrm{I} .055(0.52 \mathrm{I}, 2.135)$ & $0.863(0.405,1.842)$ & $0.762(0.359,1.615)$ & 0.823 \\
\hline Model 2 & I & $1.010(0.487,2.095)$ & $0.942(0.432,2.052)$ & $0.755(0.348,1.637)$ & 0.869 \\
\hline
\end{tabular}

Notes: Model I, without adjustment; Model 2, adjustment for age, pre-pregnancy BMI, education, smoking status, alcohol status, parity, conception method. Abbreviation: GDM, gestational diabetes mellitus. 
Table 4 ORs $(95 \% \mathrm{Cl})$ for the Occurrence of GDM According to the Quartiles of Urine Specific Gravity (N=1747)

\begin{tabular}{|c|c|c|c|c|c|}
\hline & \multicolumn{4}{|c|}{ Quartiles of Specific Gravity of Urine } & \multirow[t]{2}{*}{$P$ Trend } \\
\hline & QI & Q2 & Q3 & Q4 & \\
\hline \multicolumn{6}{|l|}{ Total } \\
\hline $\mathrm{N}$ (case/control) & $66 / 367$ & $107 / 342$ & $111 / 317$ & $110 / 327$ & \\
\hline Model I & I & $1.740(1.238,2.445)$ & $1.947(1.386,2.735)$ & $1.871(1.332,2.627)$ & $<0.001$ \\
\hline Model 2 & I & $1.74 \mid(1.233,2.458)$ & $1.863(1.319,2.633)$ & $1.703(1.203,2.41 \mathrm{I})$ & 0.004 \\
\hline \multicolumn{6}{|c|}{ Stratified analysis by age } \\
\hline \multicolumn{6}{|l|}{$\leq 35$ years } \\
\hline $\mathrm{N}$ (case/control) & $50 / 307$ & $80 / 297$ & $89 / 264$ & $90 / 269$ & \\
\hline Model I & I & $\mathrm{I} .654(\mathrm{I} .13 \mathrm{I}, 2.484)$ & $2.01 \mathrm{I}(\mathrm{I} .36 \mathrm{I}, 2.97 \mathrm{I})$ & $1.878(1.269,2.778)$ & 0.003 \\
\hline Model 2 & I & $1.676(1.090,2.421)$ & $2.124(1.446,3.119)$ & $1.945(1.314,2.880)$ & 0.001 \\
\hline \multicolumn{6}{|l|}{$>35$ years } \\
\hline $\mathrm{N}$ (case/control) & $16 / 60$ & $27 / 45$ & $22 / 53$ & $20 / 58$ & \\
\hline Model I & I & $2.250(1.085,4.665)$ & I.557 (0.74I, 3.270) & $1.293(0.6 \mathrm{II}, 2.737)$ & 0.158 \\
\hline Model 2 & I & $1.229(\mathrm{I} .06 \mathrm{I}, 4.682)$ & $1.475(0.689,3.159)$ & $1.210(0.560,2.615)$ & 0.159 \\
\hline
\end{tabular}

Notes: Model I, without adjustment; Model 2, adjustment for age, pre-pregnancy BMI, education, smoking status, alcohol status, parity, conception method. Abbreviation: GDM, gestational diabetes mellitus.

insulin use in patients who already have diabetes. ${ }^{37}$ Meanwhile, patients with primary aldosteronism were reported to be more likely to have DM due to increased UN levels resulting from impaired renal function, relative to the general population. ${ }^{38}$ A study in China by Feng et al that included 13,448 eligible pregnant women, of which 2,793 had GDM, reported that elevated UN levels in early pregnancy were positively and dose-responsively correlated with an increased risk of GDM. ${ }^{22}$ However, we detected no association between UN and GDM in either our total population or age-stratified subgroups. The main difference between the study by Feng et $\mathrm{al}^{22}$ and the present study is the age of the participants (27.63 \pm 4.09 vs $31.55 \pm 3.91$ years, respectively), which may provide a potential explanation for the discrepancy between the studies, particularly due to our finding that

Table 5 ORs $(95 \% \mathrm{Cl})$ for the Occurrence of GDM According to the Quartiles of Composite Biomarker Score (N=I569)

\begin{tabular}{|c|c|c|c|c|c|}
\hline & \multicolumn{4}{|c|}{ Quartiles of Composite Biomarker Score } & \multirow[t]{2}{*}{$P$ Trend } \\
\hline & QI & Q2 & Q3 & Q4 & \\
\hline \multicolumn{6}{|l|}{ Total } \\
\hline $\mathrm{N}$ (case/control) & $63 / 329$ & $80 / 312$ & $99 / 294$ & $112 / 280$ & \\
\hline Model I & I & $1.339(0.930,1.928)$ & $1.759(1.236,2.503)$ & $2.089(1.476,2.957)$ & $<0.001$ \\
\hline Model 2 & I & $1.342(0.926,1.945)$ & $1.68 I(1.17 I, 2.413)$ & $1.909(1.332,2.736)$ & 0.003 \\
\hline \multicolumn{6}{|c|}{ Stratified analysis by age } \\
\hline \multicolumn{6}{|l|}{$\leq 35$ years } \\
\hline $\mathrm{N}$ (case/control) & $41 / 272$ & $66 / 265$ & $78 / 254$ & $90 / 228$ & \\
\hline Model I & I & $1.652(1.080,2.527)$ & $2.037(1.345,3.085)$ & $2.619(1.740,3.942)$ & $<0.001$ \\
\hline Model 2 & I & $1.629(1.057,2.509)$ & $1.936(1.266,2.962)$ & $2.424(1.590,3.694)$ & 0.001 \\
\hline \multicolumn{6}{|l|}{$>35$ years } \\
\hline $\mathrm{N}$ (case/control) & $22 / 57$ & $14 / 47$ & $21 / 40$ & $22 / 52$ & \\
\hline Model I & I & $0.772(0.356,1.672)$ & $1.360(0.66 \mathrm{I}, 2.799)$ & $1.096(0.544,2.208)$ & 0.570 \\
\hline Model 2 & 1 & $0.755(0.344,1.657)$ & $1.258(0.597,2.652)$ & $0.942(0.449,1.974)$ & 0.670 \\
\hline
\end{tabular}

Notes: Model I, without adjustment; Model 2, adjustment for age, pre-pregnancy BMI, education, smoking status, alcohol status, parity, conception method. Abbreviation: GDM, gestational diabetes mellitus. 
increased maternal age may have an impact on the likelihood of developing GDM in response to altered kidney function.

We initially explored the relationship between USG and GDM. USG depends on the amount of dissolved substances (namely urea and sodium chloride) in the urine, and thus reflects the clearance capacity of the kidney. A mouse model of chronic kidney disease (CKD) reported that elevated circulating urea levels can increase islet protein $O$-GlcNAcylation, thereby impairing glycolysis and ultimately leading to CKD-related insulin secretion defects. ${ }^{14}$ At present, human studies demonstrating the ability of altered USG to affect glucose metabolism are still emerging.

Our study comprehensively explored the associations of three parameters used to evaluate renal function during the 16-18th week of gestation with the risk of GDM in a relatively large sample. However, some limitations remain. First, the small size of the subgroup of women aged $>35$ years limited the statistical power. Second, the retrospective design of this study may have led to inaccurate/missing data for some variables. Third, residual confounding biases may remain due to unmeasured or unknown variables. For example, a previous study demonstrated that gestational weight gain was an essential factor in determining the incidence of GDM. ${ }^{39}$ Baseline $\mathrm{FPG},{ }^{40}$ hemoglobin, ${ }^{41}$ and $\mathrm{BP}^{42}$ might also be potential confounders for GDM. However, owing to the lack of data on these parameters, we did not include them as covariates in our adjusted model. Fourth, as all participants in our study were Chinese, caution should be exercised when extrapolating these findings to other global populations.

\section{Conclusions}

In conclusion, higher UA and USG levels and a higher composite biomarker score of kidney function during the 16-18th week of gestation were positively and independently associated with an increased risk of GDM. These results suggest that the occurrence of GDM can be predicted by biomarkers of kidney function during early pregnancy.

\section{Abbreviations}

UA, Uric acid; UN, Urea nitrogen; USG, Urine specific gravity; GDM, Gestational diabetes mellitus; OR, Odds ratio; CI, Confidence interval; OGTT, Oral glucose tolerance test; BMI, Body mass index; SD, Standard deviations; NO, Nitric oxide; CKD, Chronic kidney disease.

\section{Acknowledgments}

The authors would like to acknowledge all the study participants and the clinical staff at Union Shenzhen Hospital of Huazhong University of Science and Technology for their support and contribution to this project.

\section{Funding}

This study was supported by Shenzhen Science and Technology Innovation Committee, Key Project of Shenzhen Nanshan Science and Technology Program Project funds (No.JCYJ20190809102203602), Guangdong Basic and Applied Basic Project (No.2019A1515110456) and The Maternal and Children Nutrition and Care Fund of Biostime (No.2019BINCMCF124). We thank all the study participants and the clinical staff at Union Shenzhen Hospital of Huazhong University of Science and Technology for their support and contribution to this project.

\section{Disclosure}

The authors have no conflicts of interest to declare.

\section{References}

1. Buchanan TA, Xiang AH. Gestational diabetes mellitus. $J$ Clin Invest. 2005;115(3):485-491. doi:10.1172/JCI24531

2. Zhu Y, Zhang C. Prevalence of gestational diabetes and risk of progression to type 2 diabetes: a global perspective. Curr Diab Rep. 2016;16(1):7. doi:10.1007/s11892-015-0699-x

3. Gao C, Sun X, Lu L, Liu F, Yuan J. Prevalence of gestational diabetes mellitus in mainland China: a systematic review and meta-analysis. J Diabetes Investig. 2019;10(1):154-162. doi:10.1111/jdi.12854

4. Muche AA, Olayemi OO, Gete YK. Effects of gestational diabetes mellitus on risk of adverse maternal outcomes: a prospective cohort study in Northwest Ethiopia. BMC Pregnancy Childbirth. 2020;20 (1):73. doi:10.1186/s12884-020-2759-8

5. Yogev Y, Xenakis EM, Langer O. The association between preeclampsia and the severity of gestational diabetes: the impact of glycemic control. Am J Obstet Gynecol. 2004;191(5):1655-1660. doi:10.1016/j. ajog.2004.03.074

6. Jensen DM, Sørensen B, Feilberg-Jørgensen N, Westergaard JG, BeckNielsen H. Maternal and perinatal outcomes in 143 Danish women with gestational diabetes mellitus and 143 controls with a similar risk profile. Diabet Med. 2000;17(4):281-286. doi:10.1046/j.14645491.2000.00268.x

7. Wendland EM, Torloni MR, Falavigna M, et al. Gestational diabetes and pregnancy outcomes-a systematic review of the World Health Organization (WHO) and the international association of diabetes in pregnancy study groups (IADPSG) diagnostic criteria. $B M C$ Pregnancy Childbirth. 2012;12:23. doi:10.1186/1471-2393-12-23

8. Farrar D, Simmonds M, Bryant M, et al. Hyperglycaemia and risk of adverse perinatal outcomes: systematic review and meta-analysis. BMJ. 2016;354:i4694. doi:10.1136/bmj.i4694

9. Johns EC, Denison FC, Norman JE, Reynolds RM. Gestational diabetes mellitus: mechanisms, treatment, and complications. Trends Endocrinol Metab. 2018;29(11):743-754. doi:10.1016/j. tem.2018.09.004 
10. Bellamy L, Casas JP, Hingorani AD, Williams D. Type 2 diabetes mellitus after gestational diabetes: a systematic review and meta-analysis. Lancet. 2009;373(9677):1773-1779. doi:10.1016/ S0140-6736(09)60731-5

11. Kim SY, Sharma AJ, Callaghan WM. Gestational diabetes and childhood obesity: what is the link? Curr Opin Obstet Gynecol. 2012;24 (6):376-381. doi:10.1097/GCO.0b013e328359f0f4

12. Kang DH, Nakagawa T. Uric acid and chronic renal disease: possible implication of hyperuricemia on progression of renal disease. Semin Nephrol. 2005;25(1):43-49. doi:10.1016/j.semnephrol.2004.10.001

13. Seki M, Nakayama M, Sakoh T, et al. Blood urea nitrogen is independently associated with renal outcomes in Japanese patients with stage 3-5 chronic kidney disease: a prospective observational study. BMC Nephrol. 2019;20(1):115. doi:10.1186/s12882-019-1306-1

14. Koppe L, Nyam E, Vivot K, et al. Urea impairs $\beta$ cell glycolysis and insulin secretion in chronic kidney disease. J Clin Invest. 2016;126 (9):3598-3612. doi:10.1172/JCI86181

15. Modan M, Halkin H, Karasik A, Lusky A. Elevated serum uric acida facet of hyperinsulinaemia. Diabetologia. 1987;30(9):713-718. doi:10.1007/BF00296994

16. Lv Q, Meng XF, He FF, et al. High serum uric acid and increased risk of type 2 diabetes: a systemic review and meta-analysis of prospective cohort studies. PLoS One. 2013;8(2):e56864. doi:10.1371/journal.pone. 0056864

17. Şahin Aker S, Yüce T, Kalafat E, Seval M, Söylemez F. Association of first trimester serum uric acid levels gestational diabetes mellitus development. Turk J Obstet Gynecol. 2016;13(2):71-74. doi:10.4274/ tjod.69376

18. Wolak T, Sergienko R, Wiznitzer A, Paran E, Sheiner E. High uric acid level during the first 20 weeks of pregnancy is associated with higher risk for gestational diabetes mellitus and mild preeclampsia. Hypertens Pregnancy. 2012;31(3):307-315. doi:10.3109/ 10641955.2010.507848

19. Güngör ES, Danişman N, Mollamahmutoğlu L. Relationship between serum uric acid, creatinine, albumin and gestational diabetes mellitus. Clin Chem Lab Med. 2006;44(8):974-977. doi:10.1515/ CCLM.2006.173

20. D'Apolito M, Du X, Zong H, et al. Urea-induced ROS generation causes insulin resistance in mice with chronic renal failure. $J$ Clin Invest. 2010;120(1):203-213. doi:10.1172/JCI37672

21. Xie Y, Bowe B, Li T, Xian H, Yan Y, Al-Aly Z. Higher blood urea nitrogen is associated with increased risk of incident diabetes mellitus. Kidney Int. 2018;93(3):741-752. doi:10.1016/j. kint.2017.08.033

22. Feng P, Wang G, Yu Q, Zhu W, Zhong C. First-trimester blood urea nitrogen and risk of gestational diabetes mellitus. $J$ Cell Mol Med. 2020;24(4):2416-2422. doi:10.1111/jcmm.14924

23. Behrend EN, Botsford AN, Mueller SA, Hofmeister EH, Lee HP. Effect on urine specific gravity of the addition of glucose to urine samples of dogs and cats. Am J Vet Res. 2019;80(10):907-911. doi:10.2460/ajvr.80.10.907

24. Hiersch L, Berger H, Okby R, et al. Incidence and risk factors for gestational diabetes mellitus in twin versus singleton pregnancies. Arch Gynecol Obstet. 2018;298(3):579-587. doi:10.1007/s00404018-4847-9

25. Fischer RL, Bianculli KW, Hediger ML, Scholl TO. Maternal serum uric acid levels in twin gestations. Obstet Gynecol. 1995;85 (1):60-64. doi:10.1016/0029-7844(94)00343-C

26. International Association of Diabetes and Pregnancy Study Groups Consensus Panel. International association of diabetes and pregnancy study groups recommendations on the diagnosis and classification of hyperglycemia in pregnancy. Diabetes Care. 2010;33(3):676-682. doi: $10.2337 / \mathrm{dc} 09-1848$
27. McIntyre HD, Catalano P, Zhang C, Desoye G, Mathiesen ER, Damm P. Gestational diabetes mellitus. Nat Rev Dis Primers. 2019;5(1):47. doi:10.1038/s41572-019-0098-8

28. Zhi L, Yuzhang Z, Tianliang H, Hisatome I, Yamamoto T, Jidong C. High uric acid induces insulin resistance in cardiomyocytes in vitro and in vivo. PLoS One. 2016;11(2):e0147737. doi:10.1371/journal. pone. 0147737

29. Nakagawa T, Tuttle KR, Short RA, Johnson RJ. Hypothesis: fructose-induced hyperuricemia as a causal mechanism for the epidemic of the metabolic syndrome. Nat Clin Pract Nephrol. 2005;1 (2):80-86. doi:10.1038/ncpneph0019

30. Roy D, Perreault M, Marette A. Insulin stimulation of glucose uptake in skeletal muscles and adipose tissues in vivo is NO dependent. $\mathrm{Am}$ J Physiol. 1998;274(4):E692-E699. doi:10.1152/ajpendo.1998.274.4. E692

31. Lind T, Godfrey KA, Otun H, Philips PR. Changes in serum uric acid concentrations during normal pregnancy. $\mathrm{Br} J$ Obstet Gynaecol. 1984;91(2):128-132. doi:10.1111/j.1471-0528.1984.tb05895.x

32. Laughon SK, Catov J, Provins T, Roberts JM, Gandley RE. Elevated first-trimester uric acid concentrations are associated with the development of gestational diabetes. Am J Obstet Gynecol. 2009;201 (4):402.e1-402.e4025. doi:10.1016/j.ajog.2009.06.065

33. Maged AM, Moety GA, Mostafa WA, Hamed DA. Comparative study between different biomarkers for early prediction of gestational diabetes mellitus. J Matern Fetal Neonatal Med. 2014;27 (11):1108-1112. doi:10.3109/14767058.2013.850489

34. DeBoer MD, Dong L, Gurka MJ. Racial/ethnic and sex differences in the relationship between uric acid and metabolic syndrome in adolescents: an analysis of national health and nutrition survey 1999-2006. Metabolism. 2012;61(4):554-561. doi:10.1016/j. metabol.2011.09.003

35. Park MH, Kim DH, Lee EK, et al. Age-related inflammation and insulin resistance: a review of their intricate interdependency. Arch Pharm Res. 2014;37(12):1507-1514. doi:10.1007/s12272-014-04746

36. Koppe L, Pelletier CC, Alix PM, et al. Insulin resistance in chronic kidney disease: new lessons from experimental models. Nephrol Dial Transplant. 2014;29(9):1666-1674. doi:10.1093/ndt/gft435

37. Xie Y, Bowe B, Li T, Xian H, Al-Aly Z. Blood urea nitrogen and risk of insulin use among people with diabetes. Diab Vasc Dis Res. 2018;15(5):409-416. doi:10.1177/1479164118785050

38. Liu Y, Zhou L, Liu Z, et al. Higher blood urea nitrogen and urinary calcium: new risk factors for diabetes mellitus in primary aldosteronism patients. Front Endocrinol (Lausanne). 2020;11:23. doi:10.3389/ fendo.2020.00023

39. Lewandowska M, Więckowska B, Sajdak S. Pre-pregnancy obesity, excessive gestational weight gain, and the risk of pregnancy-induced hypertension and gestational diabetes mellitus. J Clin Med. 2020;9 (6):1980. doi:10.3390/jcm9061980

40. Sesmilo G, Prats P, Garcia S, et al. First-trimester fasting glycemia as a predictor of gestational diabetes (GDM) and adverse pregnancy outcomes. Acta Diabetol. 2020;57(6):697-703. doi:10.1007/s00592019-01474-8

41. Wang C, Lin L, Su R, et al. Hemoglobin levels during the first trimester of pregnancy are associated with the risk of gestational diabetes mellitus, pre-eclampsia and preterm birth in Chinese women: a retrospective study. BMC Pregnancy Childbirth. 2018;18 (1):263. doi:10.1186/s12884-018-1800-7

42. Hedderson MM, Ferrara A. High blood pressure before and during early pregnancy is associated with an increased risk of gestational diabetes mellitus. Diabetes Care. 2008;31(12):2362-2367. doi: $10.2337 / \mathrm{dc} 08-1193$ 


\section{Publish your work in this journal}

Diabetes, Metabolic Syndrome and Obesity: Targets and Therapy is an international, peer-reviewed open-access journal committed to the rapid publication of the latest laboratory and clinical findings in the fields of diabetes, metabolic syndrome and obesity research. Original research, review, case reports, hypothesis formation, expert opinion and commentaries are all considered for publication. The manuscript management system is completely online and includes a very quick and fair peer-review system, which is all easy to use. Visit http://www.dovepress.com/testimonials.php to read real quotes from published authors. 